\title{
Vorwort zur zwölften Auflage
}

In der neuen zwölften Auflage habe ich mich bemüht, das Buch durch Nachträge und Ergänzungen auf den neuesten Stand zu bringen.

Köln, im Juni 1950

Heinrich Lehmann

\section{Vorwort zur fünften Auflage}

Der Allgemeine Teil des Bürgerlichen Gesetzbuches ist wieder in seine alten Rechte eingesetzt worden. Damit werden die Wünsche aller derer erfüllt, die das Bedürfnis empfinden, die Fülle des privatrechtlichen Stoffes von einem höheren Blickpunkt aus zu übersehen, die leitenden Grundgedanken herauszuarbeiten und $\mathrm{zu}$ einem einheitlichen Bau $\mathrm{zu}$ ordnen - also namentlich die Wünsche der Studenten, die bei dem Rechtsstudium nach der aufgehobenen nazistischen Studienordnung über mangelnden Überblick klagten, aber auch die Wünsche der fertigen Juristen, denen die Rechtsanwendung mehr als eine handwerksmäßige Fertigkeit in der Handhabe der einzelnen Gesetzesbestimmungen bedeutet.

Die Bedenken, die man in dem letzten Jahrzwölft gegen den Allgemeinen Teil geltend gemacht hat, lassen sich keinenfalls gegen die wissenschaftliche Herausarbeitung der Grundsätze erbeben, die ein Rechtsgebiet beherrschen, sondern allenfalls gegen die gesetzliche Festlegung solcher Grundsätze durch vorzeitig verallgemeinerte Begriffe, also ehe das gesamte Tatsachenmaterial, für das sie in Betracht kommen, klar angeschaut und abgegrenzt ist. Das ist allerdings im Kernstück des Allgemeinen Teils, dem Abschnitt über Rechtsgeschäfte ( $\$ \$ 104-185$ BGB.), nicht überall hinreichend beachtet worden. Es ist eine Schwäche des Allgemeinen Teils, daß seine Vorschriften über die Rechtsgeschäfte, obwohl sie z. T. nur Abstraktionen aus den angeschauten Tatbeständen des individualrechtlichen Verkehrs unter Einzelpersonen sind, gleichwohl ihrem Wortlaut nach allgemeine Anforderungen für alle Rechtsgeschäfte enthalten. So hat sich in den verflossenen 
Jahrzehnten immer mehr herausgestellt, daß diese Vorschriften der Eigenart der sozialrechtlichen Geschäfte, wie z. B. der des Verlöbnisses, der Gesellschaftsgründung, der Beitrittserklärung zur Gesellschaft, Beschluß und Abstimmung usw. nicht hinreichend gerecht werden. Das hat den Nachteil mit sich gebracht, daß ein vom Gesetzgeber in seiner Wesensart noch nicht richtig gewerteter sozialrechtlicher Tatbestand unter eine nicht passende Vorschrift gebracht wurde. Das wurde besonders bei der Einkleidung des Verlöbnisses in die Form eines "Vertrages" im Sinne des Allgemeinen Teils durch das Reichsgericht (RG. 61270 u. 80 89) klar. Der Rechtsprechung fällt es erfahrungsgemäß viel schwerer, Restriktion zu treiben als sich zur Analogie zu entschließen, obwohl jene als ein Engerdenken der Norm aus dem vernünftigen Gesetzeszweck heraus genauso berechtigt und notwendig ist als diese, die den im Gesetz niedergelegten Grundgedanken für die nicht geregelten, aber gleich zu behandelnden Tatbestände zu Ende denkt.

Die mit den Abstraktionen des Allgemeinen Teils verbundene Gefahr läßt sich durch eine richtige Gesetzesanwendung mit diesen beiden Mitteln durchaus bannen, wenn die Rechtsprechung der Aufgabe gerecht wird, jeder Anwendung einer gesetzlichen Vorschrift eine sorgfältige Untersuchung der durch sie getroffenen Tatsachen und Lebensverhältnisse sowie des vernünftig begrenzten oder erstrebten Gesetzeszweckes vorangehen zu lassen. Das Gesetz will im Zweifel die vernünftige und gerechte Ordnung der Lebensverhältnisse evident machen, nicht aber diese unpassend zurechtstutzen.

In diesem Sinne verstanden und gehandhabt, verlieren die Vorschriften des Allgemeinen Teils das Bedenkliche zu weit getriebener Abstraktion. Statt ein Prokrustesbett der Lebenstatbestände werden sie ein unvergleichliches Mittel zur Beherrschung, systematischen Ordnung und gerechten Handhabung der positiven Rechtsnormen.

Köln, Weihnachten 1946 


\section{Vorwort zur ersten Auflage}

Der "Allgemeine Teil" des Bürgerlichen Gesetzbuches gibt keine inhaltliche Regelung der einzelnen Lebens-und Rechtsverhältnisse. Er enthält nichts darüber, wie z. B. Käufe oder Mietverträge abgeschlossen werden und wirken, wie man Eigentum erwirbt, die Ehe eingeht oder ein Testament macht usw.

Aber alle diese einzelnen Rechtsvorgänge und die dadurch begründeten Rechtsverhältnisse haben gemeinsame Bestandteile. Großen Einfluß hat beim Rechtserwerb und -verlust z. B. die Willenserklärung: Käufer und Mieter wird man grundsätzlich nur, wenn man eine darauf gerichtete Willenserklärung abgibt, ebenso ist es grundsätzlich bei Eigentumserwerb, Schließung der Ehe, Ernennung des Erben. Wichtigster Gegenstand rechtlicher Herrschaft sind die Sachgüter. Utberall ist die Frage bedeutsam, wem die Rechte zustehen können: nur natürlichen Personen oder auch sonstigen menschlichen Einrichtungen wie Vereinen oder Stiftungen? Zudem eignet sämtlichen Rechten ein gleichmäßiges Grundgefüg $\theta$.

So liegt es nahe, diese gemeinsamen Bestandteile auszuscheiden und der inhaltlichen Regelung der einzelnen Rechtsverhältnisse in einem allgemeinen Teil voranzustellen. Dadurch werden nicht bloß Wiederholungen vermieden, sondern es wird auch erst die geistige Beherrschung des Rechtsstoffs gewonnen. Selbst wenn das Gesetz unterlassen hätte, die Bildung solcher Grundbegriffe und Obersätze vorzunehmen, so müBte die Wis s en s chaft diese Arbeit leisten. Denn sie hat die Aufgabe, die Fülle des Rechtsstoffs unter letzte einheitliche Oberbegriffe zu bringen und das geistige Band zu knüpfen, das die Teile zu einem einheitlichen Ganzen zusammenfaßt. So haben auch schon die Lehrbücher des Gemeinen Rechts den einzelnen Rechtsgebieten einen allgemeinen Teil vorausgeschickt. Demgegenüber ist beim Bürgerlichen Gesetzbuch das Neue nur, daß das Gesetz dieses Verfahren angenommen hat und selber in einem ersten Buche, dem sog. „Allgemeinen Teil“, seine Oberbegriffe und Leitsätze formt.

Der ,Allgemeine Teil“ bringt danach keine völligen Neuwerte, sondern enthält zum größten Teil altes Gedankengut, das Ergebnis jahrhundertelanger Gelehrtenarbeit. Abhängig ist er von der Vergangenheit schon in seinem Aufbau, der an die alte römische Dreiteilung erinnert, in das ius quod pertinet ad personas, ad res und ad actiones. 
D i es er Grundriß gliedert sich teilweise anders als das Gesetz, namentlich wo es galt, das Verständnis des Anfängers nicht allzusehr zu erschweren; so ist z. B. das Personenrecht an den Schluß gestellt, wo es $\mathrm{m}$. E. auch in der Vorlesung behandelt werden muß: es setzt die Kenntnis der Lehre vom Rechtsgeschäft voraus. Im Gesetzbuch fehlen Grundsätze über die Rechtsvorschriften selbst: sie sind in einer Einleitung als "Lehre vom objektiven Recht" kurz zusammengestellt.

Auf der andern Seite schien mir nicht ratsam, einen völligen Neubau im Gefüge des Allgemeinen Teils zu versuchen, so sehr auch die Aufgabe lockt und dereinst erfüllt werden muß. Aber jetzt ist die Zeit dafür noch nicht reif und dann warnt auch vor allzu eingreifenden Neuerungen der Zweck des Grundrisses.

Immerhin mögen die von mir beliebten Abweichungen sowie die gelegentlichen abwertenden Betrachtungen den Leser davor bewahren, Begriffsgebilde und gedanklichen Aufbau des "Allgemeinen Teils" zu überschätzen. Seine Begriffe sind keine allgemeingültigen Werte, keine unveränderlichen, bloß rechnerischen Größen, die man einfach auf die einzelnen Lebens- und Rechtsverhältnisse anzuwenden brauchte, um ihre Regelung als eine logisch-mathematische Lösung wie von einem Zähler abzulesen, sie sind selber erst aus den Einzelsätzen des geltenden Rechts abgeleitet und bedürfen deshalb steter Überprüf ung nach ihrer Wirkung im Leben.

Schon der Anfänger muß sich recht frühzeitig darüber klar werden: Die Kunst des Juristen besteht nicht darin, die Rechtssätze von a u Ben an die Dinge heranzubringen und ihren gesunden Lebenswuchs zurechtzustutzen, sondern das Recht aus den angeschauten Dingen, aus der greifbaren Wirklichkeit des frischen Lebens herauszuholen; jeder Begriff ist so auszulegen, daß er einen möglichst hohen Lebenswert hat, die Gerechtigkeit darbietet, die menschlichem Können erreichbar ist. Möglichst gerecht den Fall entscheiden, das ist in allem das Leitziel. 